\title{
Análise micromorfológica de esmalte e dentina expostos ao laser de Nd:YAG em regime de picossegundos
}

\section{Micro-morphological analisys of enamel and dentin exposed to picosecond-pulsed Nd:YAG laser}

\author{
Rosane de Fátima Zanirato Lizarelli* \\ Vanderlei Salvador Bagnato**
}

\begin{abstract}
RESUMO: A eficiência da ablação com laser através de um regime de picossegundos minimiza a destruição do material adjacente devido à formação de plasma e ataque. Isso previne a geração excessiva de ondas de choque e promove uma considerável diminuição nos efeitos mecânicos. Nesse estudo foi utilizado um laser de Nd:YAG em picossegundo "Q-switched" e "mode-locked" (Antares 76-s, Coherent, Palo Alto, CA), três molares humanos recém-extraídos e foram realizados 15 microcavidades em esmalte e 9 em dentina. Os parâmetros para irradiar o esmalte seguiram uma freqüência fixa de $100 \mathrm{~Hz}$ e potência média de $1,3 \mathrm{~W}$; enquanto que para a dentina, a freqüência foi fixa em $15 \mathrm{~Hz}$ enquanto que a potência média variou em 800,700 e $600 \mathrm{~mW}$. O esmalte demonstrou ser mais capaz de dissipar as ondas de choque do que a dentina, uma vez que as trincas originadas nas microcavidades foram mais freqüentes em tecido dentinário, diferentemente do esmalte. Em conclusão, parece que para manter os aspectos de definição de bordas é importante escolher tempos de irradiação mais curtos, quando as potências médias forem mantidas mais altas, ou seja, energia total entregue mais baixa e bem localizada.
\end{abstract}

UNITERMOS: Laser; Nd:YAG; Esmalte; Dentina.

\begin{abstract}
The efficiency of the ablation obtained with a picosecond-pulsed laser system minimizes material collateral damage because of the etching and plasma formation mechanism. It prevents the excessive generation of shock waves and promotes a considerable decrease of mechanical side effects. In this study, a Nd:YAG picosecond-pulsed Q-switched and mode-locked laser (Antares 76-s, Coherent, Palo Alto, CA) was used. Fifteen microcavities were prepared in the enamel, and nine microcavities were prepared in the dentin of three recently extracted human molars. The parameters for irradiating enamel followed a fixed frequency of $100 \mathrm{~Hz}$ and an average power level of $1.3 \mathrm{~W}$; for dentin, the frequency was fixed in $15 \mathrm{~Hz}$, and the average power level varied between 800, 700 and $600 \mathrm{~mW}$. Enamel proved more capable to dissipate shock waves than dentin, since cracks originating from microcavities were more frequent in dentin. In conclusion, it seems that in order to keep defined edges, it is important to choose shorter irradiation periods when average power levels are kept high, which results in lower and best-localized total energy delivery.

UNITERMS: Laser; Nd:YAG; Enamel; Dentin.
\end{abstract}

\section{INTRODUÇÃO}

Vários experimentos têm demonstrado que pulsos ultracurtos no domínio de subpicossegundos promovem uma combinação de efeitos termomecânicos que superam algumas das objeções ao uso de um laser como instrumento removedor. Usando os parâmetros apropriados de operação, lasers com pulsos ultracurtos podem se comportar melhor do que instrumentos convencionais, incluindo alguns dos lasers pulsados comercialmente já disponíveis na Odontologia.

Atualmente, o laser mais comumente utilizado em Odontologia opera no regime pulsado com duração de pulsos na faixa de milissegundos $(1 \mathrm{~ms}$ equivale a $10^{-6}$ segundos) e nanossegundos (1 $\mathrm{ns}$ equivale a $10^{-9}$ segundos). O laser de Nd:YAG com chaveamento Q normalmente opera em 300 ns enquanto que um laser de Nd:YAG em operação livre apresenta operação no regime pulsado de $1 \mathrm{~ms}$. Laser com pulsos de largura menor do que 1 ns é normalmente chamado de laser de pulso curto. Nesse regime de operação o principal mecanismo de ablação ocorre através da vaporização. Pulsos longos até microssegundos geram muito calor durante o processo de ablação e normalmente encontram aplicações na Odontologia com certas limitações. Danos colaterais aos tecidos ou materiais pelos efeitos térmicos estão comumente presentes. A duração do pulso é o parâmetro mais importante

${ }^{*}$ Cirurgiã-Dentista, Mestre e Doutoranda em Ciências e Engenharia de Materiais; **Doutor em Física Atômica e Docente - Instituto de Física de São Carlos da Universidade de São Paulo. 
Lizarelli R de FZ, Bagnato VS. Análise micromorfológica de esmalte e dentina expostos ao laser de Nd:YAG em regime de picossegundos. Pesqui Odontol Bras 2002;16(3):227-233.

devido ao fato de que durante esse tempo de interação a difusão de calor torna-se um importante fato no mecanismo de interação envolvendo a luz e o tecido. O excesso de calor pode promover fortes efeitos colaterais, uma vez que a temperatura no interior do órgão dental pode atingir valores superiores à $10^{\circ} \mathrm{C}$ de variação, com a grande possibilidade de danos permanentes. Encurtando os pulsos, ocorre diminuição de efeitos térmicos e introduz novos mecanismos como a ablação mediada por plasma ${ }^{2,6,9}$.

Os experimentos de ablação de tecidos dentais realizados com laser com pulsos em picossegundos foram primeiramente reportados por Niemz $z^{8}$. Neste estudo foi mostrado que lasers de Nd:YLF são alternativas viáveis para remoção de cáries. A diferença na taxa de ablação para esmalte cariado e sadio conduz a uma remoção seletiva de cárie. Nesse caso, o uso de pulsos em picossegundos claramente minimiza os efeitos colaterais normalmente observados quando lasers com pulsos longos são usados.

Pulsos curtos e ultracurtos têm um tipo de interação com o tecido através do efeito fotoelétrico. A excitação de pulsos curtos resulta em menor volume aquecido que também induz à diminuição da energia mínima necessária para ablação. Essa é a principal razão de porque se obtém uma razoável taxa de ablação para picossegundos utilizando fluências menores do que aquelas convencionais nos sistemas pulsados em nanosegundos. Um problema comum na ablação por pulsos é a formação de plasma que se localiza próxima à superfície. O plasma é formado na maioria das vezes através do processo de ionização multifotônica e acaba por bloquear a subseqüente energia luminosa incidente. O plasma formado pelos pulsos curtos é altamente energético. Esse plasma de alta energia age como um agente de ataque, removendo material pelo bombardeamento iônico à superficie. Esse mecanismo está de acordo com o fato de que a estrutura prismática do esmalte é preservada mesmo dentro da região ablacionada para o caso dos pulsos em picossegundo, ou mesmo uma boa revelação dos túbulos, removendo todo o "smear layer", no caso da dentina ${ }^{3,4}$.

Neste trabalho, foram realizadas microcavidades em esmalte e em dentina, para análise comparativa, empregando potências operativas de $600 \mathrm{~mW}$ a $1.300 \mathrm{~mW}$.

\section{MATERIAIS E MÉTODOS}

Foram utilizados três molares humanos recém-extraídos que estavam armazenados em soro fisiológico, à temperatura ambiente $\left(25^{\circ} \mathrm{C}\right)$ e foram esterilizados em autoclave imersos em água destilada em recipiente de vidro transparente vedado com papel tipo "kraft" e fita adesiva para autoclave (3M) para serem preparados. As amostras foram preparadas seguindo o seguinte protocolo: a porção radicular dos elementos foi removida com o auxílio de um disco de "carborundum" montado em peça-de-mão (Ruca, Brasil). Após, cada coroa foi seccionada no sentido ocluso-cervical, separando-se as porções vestibular e lingual. Sendo assim, os três molares resultaram em 6 metades. Essas metades foram então embutidas em um anel de PVC e resina epóxica Cristal, de forma que as faces vestibular e lingual ficassem voltadas para cima e expostas.

De posse desse corpo-de-prova, o próximo passo foi tornar as superficies vestibular e lingual planas, ao mesmo tempo em que fosse possivel a exposição de tecido de esmalte e de dentina de cada metade. Para isso, foram utilizadas lixas d'água números 80, 120, 200, 400 e 600 (Norton), nessa ordem, apoiando em bancada e usadas de forma manual.

O corpo-de-prova permaneceu armazenado em água destilada a $37^{\circ} \mathrm{C}$ até o momento do experimento (uma semana). Para remoção do biofilme superficial, as amostras foram tratadas em solução de tripsina (10 mg) e água destilada esterilizada $(10 \mathrm{ml})$ durante 10 minutos. Utilizando o sistema de translação $x-y-z$, o corpo-de-prova foi fixado no eixo com o auxílio de uma fita adesiva dupla face $(3 \mathrm{M})$. O eixo, por sua vez, foi fixado sobre a bancada onde o sistema laser estava estabilizado, através de um trilho e parafusos. Esse eixo permite a translação das amostras em 3 direções, tornando possivel e fácil trabalhar com o feixe laser focado. As amostras estavam então prontas para serem irradiadas.

Nesse estudo foi utilizado um laser de Nd:YAG pulsado no regime de picossegundo com chaveamento Q e travamento de modos (Antares 76-s, Coherent, Palo Alto, CA). Esse laser emite trens de pulsos de $70 \mathrm{ps}$ em $1.064 \mathrm{~nm}$. Cada sinal de $70 \mathrm{ps}$ é agrupado em torno de 15 pulsos intensos com intervalo de 13 ns na freqüencia controlada de repetição "Q-switch" (3-1.200 Hz). Foi utilizada uma lente com distância focal de $12,0 \mathrm{~cm}$, gerando uma área transversal do feixe de $3,85 \times 10^{-5} \mathrm{~cm}^{2}$. Esse 
Lizarelli R de FZ, Bagnato VS. Análise micromorfológica de esmalte e dentina expostos ao laser de Nd:YAG em regime de picossegundos. Pesqui Odontol Bras 2002;16(3):227-233.

TABELA 1 - Diferentes parâmetros de energia total utilizados para a confecção das microcavidades em esmalte e em dentina com laser de Nd:YAG sob regime de picossegundos.

\begin{tabular}{l|c|c|c|c|c|c|c|c|c|c|c|c}
\hline \multicolumn{1}{c|}{ Substrato } & \multicolumn{4}{c|}{ Esmalte } & \multicolumn{5}{c}{ Dentina } \\
\hline \hline Parâmetros de irradiação & \multicolumn{3}{|c|}{$1.300 \mathrm{~mW}$ e $100 \mathrm{~Hz}$} & \multicolumn{1}{c}{$800 \mathrm{~mW}$ e $15 \mathrm{~Hz}$} & \multicolumn{2}{c}{$700 \mathrm{~mW}$ e $15 \mathrm{~Hz}$} & \multicolumn{2}{c}{$600 \mathrm{~mW}$ e $15 \mathrm{~Hz}$} \\
\hline Tempos de irradiação (segundos) & 30 & 15 & 5 & 30 & 15 & 5 & 30 & 15 & 5 & 30 & 15 & 5 \\
\hline Energia total $(\mathrm{J})$ & 39 & 19,5 & 6,5 & 24 & 12 & 4 & 21 & 10,5 & 3,5 & 18 & 9 & 3 \\
\hline Fluência $\left(\times 10^{-4} \mathrm{~J} / \mathrm{cm}^{2}\right)$ & 15 & 7,5 & 2,5 & 9,24 & 4,62 & 1,54 & 8,09 & 4,04 & 1,35 & 6,93 & 3,47 & 1,16 \\
\hline Intensidade $\left(\times 10^{-6} \mathrm{~W} / \mathrm{cm}^{2}\right)$ & 15 & 7,5 & 2,5 & 61,6 & 30,8 & 10,3 & 53,9 & 26,9 & 9 & 46,2 & 23,1 & 7,73 \\
\hline \hline
\end{tabular}

laser de Nd:YAG é a fonte mais simples e a mais comum para pulsos em picossegundos, principalmente por não necessitar de um amplificador especial de pulsos.

Foram realizados 15 microcavidades em esmalte e 9 em dentina, sendo que os parâmetros para irradiar o esmalte seguiram uma freqüência fixa de $100 \mathrm{~Hz}$ e potência média de $1,3 \mathrm{~W}$; enquanto que para a dentina, a freqüência foi fixa em $15 \mathrm{~Hz}$ enquanto que a potência média variou em 800,700 e $600 \mathrm{~mW}$. Os tempos de irradiação foram escolhidos em: 30, 15 e 5 segundos. Resultando, portanto, nas seguintes energias totais $(\mathrm{J})$, fluências $\left(\mathrm{J} / \mathrm{cm}^{2}\right)$ e intensidades $\left(\mathrm{W} / \mathrm{cm}^{2}\right)$, resumidas na Tabela 1 .

\section{RESULTADOS E DISCUSSÕES}

Com finalidade didática e exploratória, consideramos as zonas e observação nas microcavidades: $Z 1$, centro da microcavidade, onde o material foi ablacionado; $Z 2$, zona intermediária que pode ou não existir; e, Z3, região do material que não recebeu influência alguma da irradiação (Figura 1).

Quando consideramos a análise comparativa da energia total entregue, dentro de cada grupo de potência média, fica claro que à medida que a energia total diminui, as bordas da microcavidade tornaram-se mais bem definidas.

Com relação à variação da potência média, também quanto menor o valor, melhor a definição das bordas da microcavidade, ou seja, menor a região $Z 2$, chegando a não ser possível, em alguns casos, sua visualização (Figuras 2C, 3B, 3C E 5C). Z2 parece resultar da esfoliação num tempo posterior ao da irradiação. Ou seja, depois de realizadas as microcavidades com 700 e com $800 \mathrm{~mW}$, a região das bordas tornou-se tão frágil a ponto de se destacar, esfoliando do tecido. Esse fenômeno pode ser conseqüência das fortes ondas de choques provocadas por essas potências mais altas.

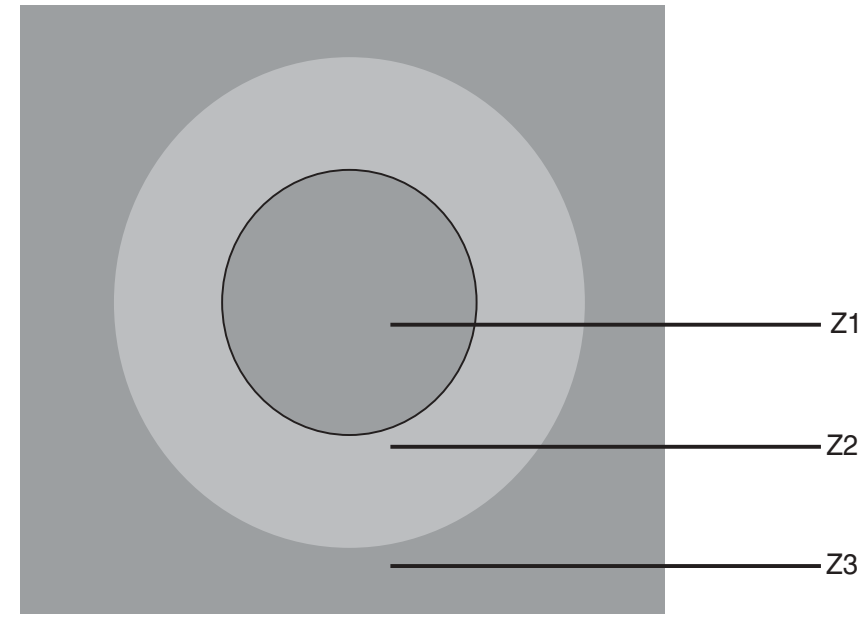

FIGURA 1 - Desenho esquemático apontando como foram classificadas as zonas morfológicas relacionadas às microcavidades. $Z 1$ = centro da microcavidade, onde $o$ material foi ablacionado; $\mathrm{Z2}=$ zona intermediária que pode ou não existir; Z3 = região do material que não recebeu influência alguma da irradiação.

Considerando o tecido-alvo, esmalte ou dentina, esse fato parece não influenciar as características morfológicas das microcavidades aqui analisadas.

Com relação ao esmalte e sem alterar a potência média, foi possivel observar que a medida que a energia total foi diminuindo, a região $Z 2$ também tendeu a desaparecer, ou seja, enquanto nas Figuras $2 \mathrm{~A}$ e $2 \mathrm{~B}$ a região intermediária está presente, descrevendo a falta de definição das bordas da microcavidade, fato diferente ocorreu na Figura 2C, onde a energia total entregue diminuiu.

Além disso, com a maior energia (Figura 2A) foi possivel observar uma grande trinca gerada tendo como centro de origem o furo produzido. Denotando que a capacidade em dissipar as ondas de choque geradas durante a irradiação pode estar associada a energia total entregue, e não à potência média empregada. Isso significa que existe um li- 
Lizarelli R de FZ, Bagnato VS. Análise micromorfológica de esmalte e dentina expostos ao laser de Nd:YAG em regime de picossegundos. Pesqui Odontol Bras 2002;16(3):227-233.
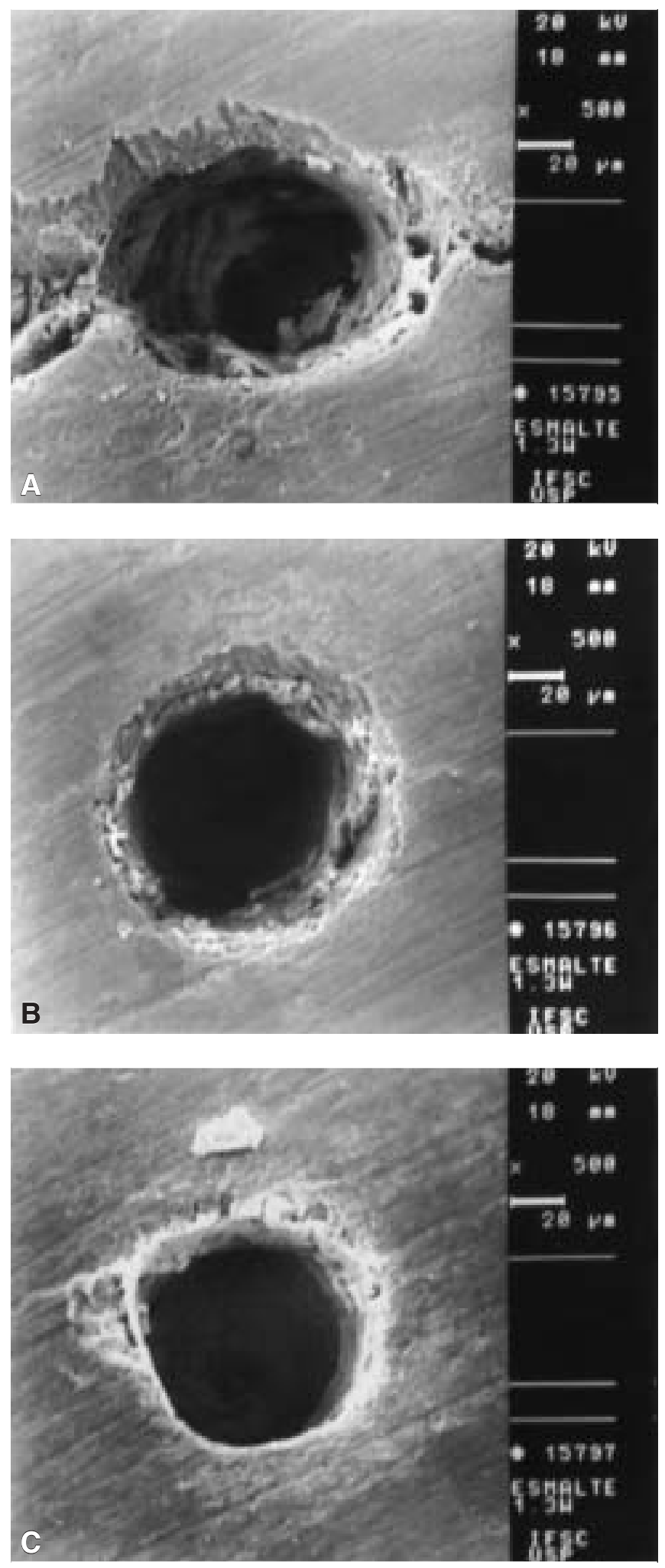

FIGURA 2 - Microcavidades em esmalte com $1.300 \mathrm{~mW}$ de potência média e tempos de exposição diferentes e decrescentes (A - 30, B - 15 e C - 5 segundos) (500 X).
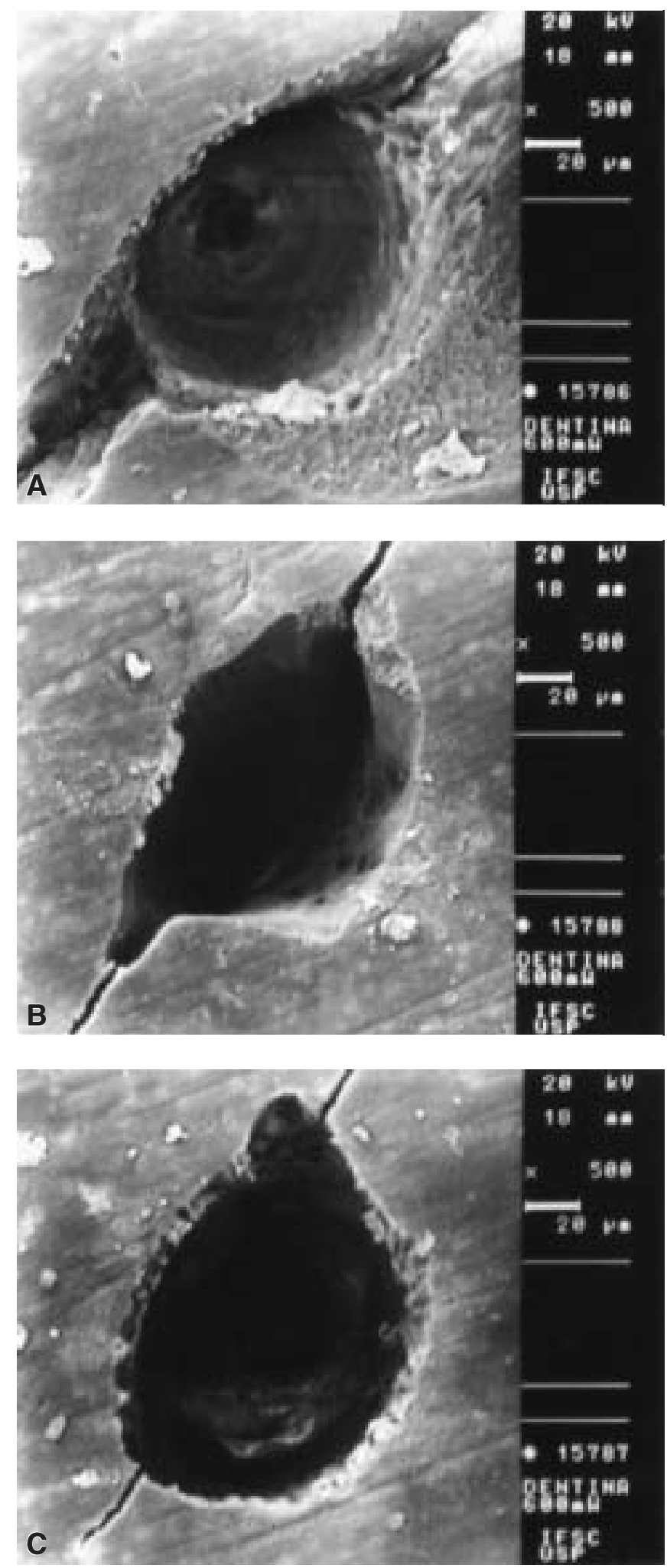

FIGURA 3 - Microcavidades em dentina com $600 \mathrm{~mW}$ de potência média e tempos de exposição diferentes e decrescentes (A - 30, B - 15 e C - 5 segundos) (500 X). 
Lizarelli R de FZ, Bagnato VS. Análise micromorfológica de esmalte e dentina expostos ao laser de Nd:YAG em regime de picossegundos. Pesqui Odontol Bras 2002;16(3):227-233.
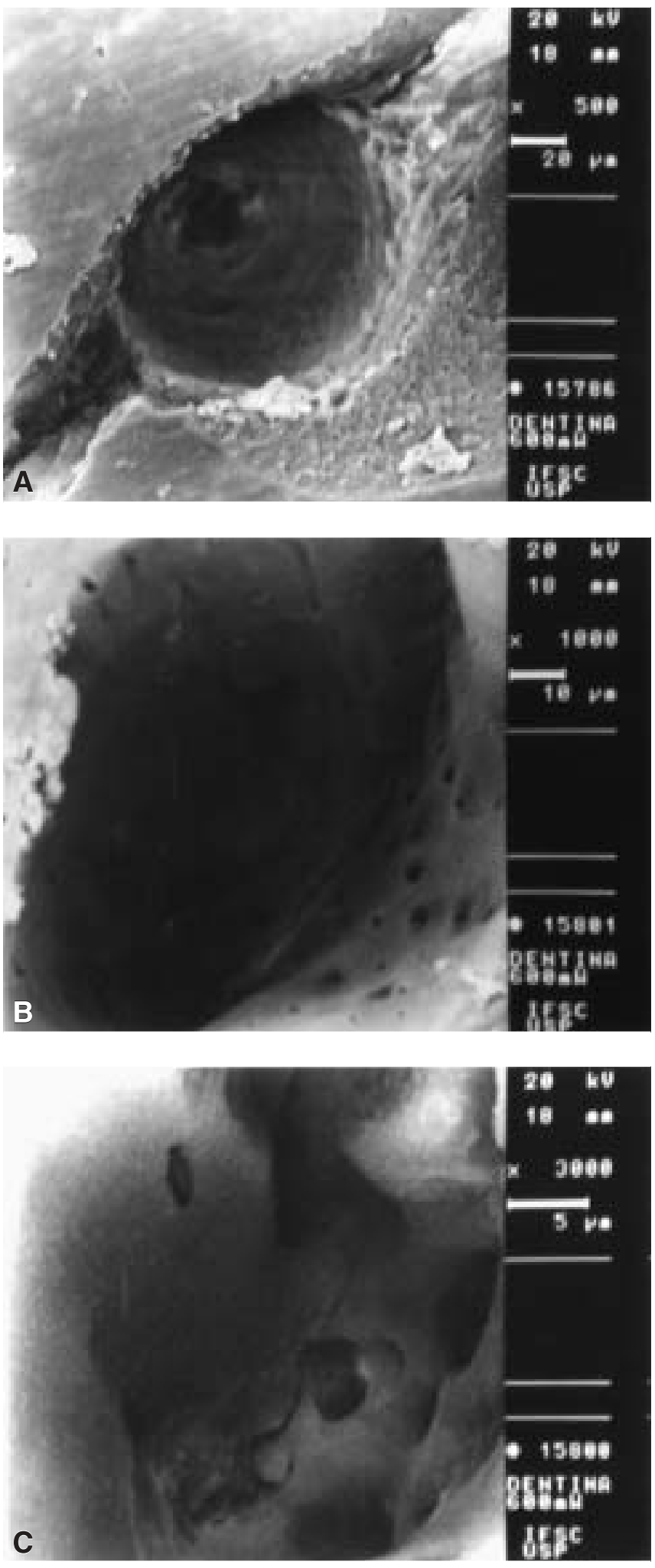

FIGURA 4 - Microcavidade em dentina com $600 \mathrm{~mW}$ de potência média e 5 segundos de tempo de exposição em três diferentes aumentos (A - 500 X, B - 1.000 X e C $3.000 \mathrm{X})$. mite também com relação à energia total empregada.

Quando são mostrados maiores aumentos dessa microcavidade com bordas mais definidas, fica novamente evidente que, de forma localizada, o laser de picossegundos promove uma fusão e ressolidificação, contudo e superficie resultante parece ser muito mais homogênea do que aquelas observadas como resultado da aplicação de laser de $\mathrm{CO}_{2}$ ou de Nd:YAG em regime pulsado em microssegundos.

Com relação ao tecido dentinário, aqui novamente torna-se notável que a menor energia total entregue foi capaz de produzir microcavidades com bordas mais definidas (Figura 3C). Contudo, como para a dentina a potência média, nesse experimento, foi variada em três valores, foi fácil perceber que a sua diminuição também acaba por influenciar a eficiência de ablação ao nível de definição de corte do laser de picossegundos. Porém, como a Figura 6C apresenta uma microcavidade sem Z2 da mesma forma como a Figura 3C, onde a potência média foi menor, novamente, parece estar lícito que a maior influência para a definição de bordas das microcavidades e ausência de zona intermediária está relacionada à energia total entregue.

Ainda com relação à dentina, foi possivel visualizar grandes trincas geradas pelas microcavidades, independente da potência média ou do tempo de exposição (Figuras 4, 5 e 6), porém sendo em tecido dentinário, esse fato não está somente relacionado ao mecanismo de interação do laser com o tecido, mas principalmente devido às características estruturais de anisotropia desse tecido dentinário.

Mesmo sob a potência mais baixa aqui empregada $(600 \mathrm{~mW})$, na Figura 4 nota-se as características térmicas resultantes da interação desse laser com o tecido dentinário, provavelmente colabando as fibras colágenas e elásticas da dentina intertubular, como pudemos constatar em outros experimentos $^{5}$, mas em algumas áreas deixando alguns túbulos dentinários abertos. Isso porque o laser de $\mathrm{Nd}$ :YAG interage mais fortemente com as porções orgânicas do tecido-alvo, vaporizando as mesmas. Como o tecido dentinário possui $30 \%$ de porção orgânica e $70 \%$ de inorgânica ${ }^{1}$, esses minerais sofrem fusão e ressolidificação, porém não resultam em quantidades suficientes para vedarem todos os túbulos dentinários durante o escoamento da fase liquida. 
Lizarelli R de FZ, Bagnato VS. Análise micromorfológica de esmalte e dentina expostos ao laser de Nd:YAG em regime de picossegundos. Pesqui Odontol Bras 2002;16(3):227-233.
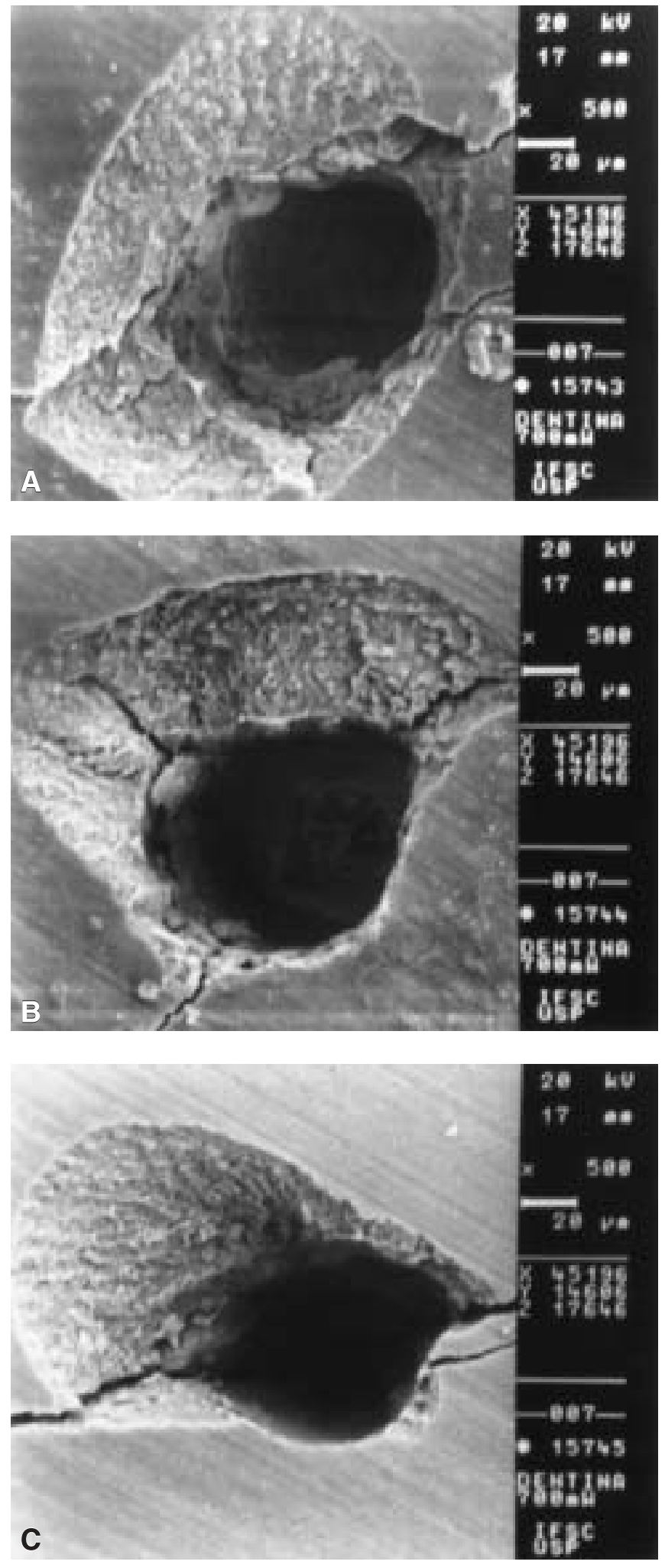

FIGURA 5 - Microcavidades em dentina com $700 \mathrm{~mW}$ de potência média e tempos de exposição diferentes e decrescentes (A - 30, B - 15 e C - 5 segundos) (500 X).
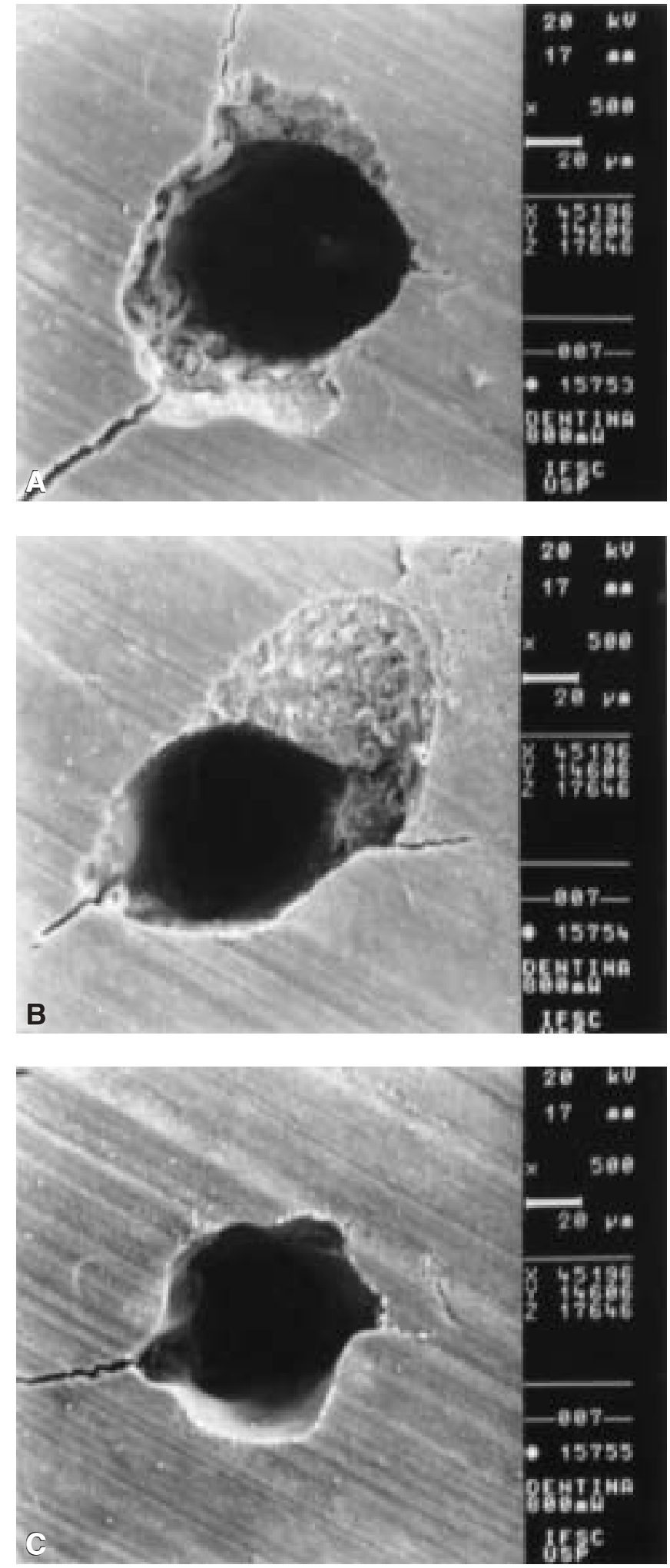

FIGURA 6 - Microcavidades em dentina com $800 \mathrm{~mW}$ de potência média e tempos de exposição diferentes e decrescentes (A - 30, B - 15 e C - 5 segundos) (500 X). 
Lizarelli R de FZ, Bagnato VS. Análise micromorfológica de esmalte e dentina expostos ao laser de Nd:YAG em regime de picossegundos. Pesqui Odontol Bras 2002;16(3):227-233.

É possivel observar que à medida que decresce a energia total entregue, também decresce o diâmetro do furo. Contudo, situação inversa ocorreu quando a potência média aumentou, ou seja, nesse caso, o diâmetro diminuiu. Parece então que é importante que a energia esteja bem concentrada no ponto focal de ablação.

\section{CONCLUSÕES}

Nesse experimento, foi possivel observar a influência da energia total entregue nos tecidos esmalte e dentina, quando potências médias mais altas foram escolhidas:

1. o esmalte demonstrou ser mais capaz de dissipar as ondas de choque do que a dentina, uma vez que as trincas originadas nas microcavidades foram mais freqüentes em tecido dentinário, diferentemente do esmalte;

\section{REFERÊNCIAS}

1. Bhaskar SN. Histologia e Embriologia Oral de Orban. Orban's oral histology 8 ed. Traduzido por Eleonora Diefenbach Muller. São Paulo: Artes Médicas, 1978.

2. Chen X, Liu X. Short-pulsed laser machining: how short is short enough? J Laser Appl 1999;11:268-72.

3. Lizarelli RFZ, Kurachi C, Misoguti L, Bagnato VS. Characterization of enamel and dentin response to Nd:YAG picosecond laser ablation. J Clin Laser Med Surg 1999;17(3):127-31.

4. Lizarelli RFZ, Kurachi C, Misoguti L, Bagnato VS. A comparative study of nano and picosecond laser ablation in enamel: morphological aspects. J Clin Laser Med Surg 2000;18(3):151-7.
2. com relação à presença de $Z 2$, quando se aumentou a potência média e também a energia total, Z2 apareceu após a irradiação devido a esfoliação das bordas fragilizadas pelas ondas de choque geradas. Esse fato se deu pela incapacidade do tecido para dissipar as ondas de choque.

Em conclusão, parece que para manter os aspectos de definição de bordas é importante escolher tempos de irradiação mais curtos, quando as potências médias forem mantidas mais altas, ou seja, energia total entregue mais baixa e bem localizada.

\section{AGRADECIMENTOS}

Aos Profs. Drs. Sérgio Carlos Zílio e Cléber Mendonça, e ao Doutorando Leonardo De Boni pela manipulação do laser de picossegundos; a CAPES.

5. Lizarelli RFZ. Lasers de Nd:YAG nos regimes de nano e picossegundos em esmalte e em dentina - análises morfológica e química. (Dissertação de Mestrado). São Carlos: Instituto de Física de São Carlos da Universidade de São Paulo, 2000.

6. Marion JE, Kim BM. Medical applications of ultra-short pulse lasers. Proceedings of SPie 1999;3910:42-50.

7. Neev J. Ultrafast lasers are on the cutting edge. Photonics Spectra 2001:44-7.

8. Niemz MH. Cavity preparation with Nd:YLF picosecond laser. J Dent Res 1995;74:1194-9.

9. Pronko PP, Dutta SK, Squier J, Rudd JV, Du D, Mourou G. Machining of sub-micron holes using a femtosecond laser at $800 \mathrm{~nm}$. Opt Commun 1995;114:106-10.

Recebido para publicação em 24/09/01 Enviado para reformulação em 29/04/02 Aceito para publicação em 15/07/02 